EUROPEAN JOURNAL OF PURE AND APPLIED MATHEMATICS

Vol. 14, No. 4, 2021, 1379-1387

ISSN 1307-5543 - ejpam.com

Published by New York Business Global

\title{
Definite integral of logarithmic power and square root algebraic functions expressed in terms of the Lerch function
}

\author{
Robert Reynolds ${ }^{1, *}$, Allan Stauffer ${ }^{2}$ \\ 1 Department of Mathematics and Statistics, Faculty of Science, York University, Toronto, \\ Ontario, Canada, MЗJ1P3
}

\begin{abstract}
A definite integral involving the power square root of an algebraic function is derived in terms of the Lerch function. A table consisting of mostly new results is given for easy reading. The majority of the results in this work are new.

2020 Mathematics Subject Classifications: Primary 30E20,33-01, 33-03, 33-04, 33-33B, $33 \mathrm{E} 20$
\end{abstract}

Key Words and Phrases: Definite integral, square root function, Lerch function, Polylogarithm function, Riemann zeta function

\section{Significance statement}

Definite integrals involving the nested square root of a function over $[2, \infty)$ have been studied by Nyblom [7]. The authors studied a definite integral with a general root kernel in [9]. In this work we will derive and evaluate integrals involving a power square root and algebraic functions over $[0, \infty)$. This integral is also derived in terms of the Lerch function which is currently not present in current literature. Possible applications of these types integrals other than those met in stochastic problems has not yet been explored.

\section{Introduction}

In the present work, the authors used their contour integral method and applied it to the Lerch function to derive a definite integral and expressed its closed form in terms of a special function. This derived integral formula was then used to provide formal derivations in terms of special functions and fundamental constants. The Lerch function being a special function has the fundamental property of analytic continuation, which enables us to widen the range of evaluation for the parameters involved in our definite integral.

*Corresponding author.

DOI: https://doi.org/10.29020/nybg.ejpam.v14i4.4065

Email addresses: milver@my.yorku.ca (R. Reynolds), stauffer@yorku.ca (A. Stauffer) 
The Lerch function is a special function that generalizes the Hurwitz zeta function, the polylogarithms, and so many interesting and important special functions. The definite integral derived in this manuscript is given by

$$
\int_{0}^{\infty} \frac{(\sqrt{b+x}-\sqrt{b})^{-m} \log ^{k}\left(\frac{a}{\sqrt{b+x}-\sqrt{b}}\right)}{x \sqrt{b+x}} d x
$$

where the parameters $k, b$ and $a$ are general complex numbers and $-1<\operatorname{Re}(m)<0$. The derivation of the definite integral follows the method used by us in [10] which involves Cauchy's integral formula. The generalized Cauchy's integral formula is given by

$$
\frac{y^{k}}{\Gamma(k+1)}=\frac{1}{2 \pi i} \int_{C} \frac{e^{w y}}{w^{k+1}} d w .
$$

where $C$ is in general an open contour in the complex plane where the bilinear concomitant [10] has the same value at the end points. This method involves using a form of equation (2) then multiply both sides by a function, then take a definite integral of both sides. This yields a definite integral in terms of a contour integral. A second contour integral is derived by multiplying equation (2) by a function and performing some substitutions so that the contour integrals are the same.

\section{Definite integral of contour integral}

We use the method in [10]. The variable of integration in the contour integral is $z=w+m$. The cut and contour are in the first quadrant of the complex $z$-plane. The cut approaches the origin from the interior of the first quadrant and the contour goes round the origin with zero radius and is on opposite sides of the cut. Using equation (2) we replace $y$ by $\frac{a}{\sqrt{b+x}-\sqrt{b}}$ then multiply by $\frac{(\sqrt{b+x}-\sqrt{b})^{-m}}{x \sqrt{b+x}}$. Next we take the infinite integral over $x \in[0, \infty)$ to get

$$
\begin{aligned}
& \frac{1}{\Gamma(k+1)} \int_{0}^{\infty} \frac{(\sqrt{b+x}-\sqrt{b})^{-m} \log ^{k}\left(\frac{a}{\sqrt{b+x}-\sqrt{b}}\right)}{x \sqrt{b+x}} d x \\
& =\frac{1}{2 \pi i} \int_{0}^{\infty} \int_{C} \frac{a^{w} w^{-k-1}(\sqrt{b+x}-\sqrt{b})^{-m-w}}{x \sqrt{b+x}} d w d x \\
& =\frac{1}{2 \pi i} \int_{C} \int_{0}^{\infty} \frac{a^{w} w^{-k-1}(\sqrt{b+x}-\sqrt{b})^{-m-w}}{x \sqrt{b+x}} d x d w \\
& =\frac{1}{2 \pi i} \int_{C} \pi a^{w} w^{-k-1}\left(-2^{-m-w}\right) b^{\frac{1}{2}(-m-w)-\frac{1}{2}} \csc (\pi(m+w)) d w
\end{aligned}
$$


from equation (2.1.8.1) in [3] where $0<\operatorname{Re}\left(\frac{w+m}{2}\right)<1,|\arg (b)|<\pi$. We are able to switch the order of integration over $w+m$ and $x$ using Fubini's theorem since the integrand is of bounded measure over the space $\mathbb{C} \times[0, \infty)$.

\section{The Lerch function}

We use equation (1.11.3) in [2] where $\Phi(z, s, v)$ is the Lerch function which is a generalization of the Hurwitz zeta $\zeta(s, v)$ and Polylogarithm functions $L i_{n}(z)$. The Lerch function has a series representation given by

$$
\Phi(z, s, v)=\sum_{n=0}^{\infty}(v+n)^{-s} z^{n}
$$

where $|z|<1, v \neq 0,-1, .$. and is continued analytically by its integral representation given by

$$
\Phi(z, s, v)=\frac{1}{\Gamma(s)} \int_{0}^{\infty} \frac{t^{s-1} e^{-v t}}{1-z e^{-t}} d t=\frac{1}{\Gamma(s)} \int_{0}^{\infty} \frac{t^{s-1} e^{-(v-1) t}}{e^{t}-z} d t
$$

where $\operatorname{Re}(v)>0$, and either $|z| \leq 1, z \neq 1, \operatorname{Re}(s)>0$, or $z=1, \operatorname{Re}(s)>1$.

\section{Infinite sum of contour integral}

In this section we will again use Cauchy's integral formula (2) and take the infinite sum to derive equivalent sum representations for the contour integrals. We proceed using equation (2) and replace $y$ by $\log (a)-\frac{\log (b)}{2}+i \pi(2 y+1)-\log (2)$ and multiply both sides by $i \pi 2^{1-m} b^{\frac{1}{2}(-m-1)} e^{i \pi m(2 y+1)}$ and take the infinite sum over $y \in[0, \infty)$ simplifying in terms of the Lerch function to get

$$
\begin{aligned}
& \frac{(i \pi)^{k+1} e^{i \pi m} b^{-\frac{m}{2}-\frac{1}{2}} 2^{k-m+1} \Phi\left(e^{2 i m \pi},-k, \frac{i(-2 \log (a)+\log (b)+\log (4)-2 i \pi)}{4 \pi}\right)}{\Gamma(k+1)} \\
& =\frac{1}{2 \pi i} \sum_{y=0}^{\infty} \int_{C} i \pi a^{w} w^{-k-1} 2^{-m-w+1} b^{\frac{1}{2}(-m-w-1)} e^{i \pi(2 y+1)(m+w)} d w \\
& =\frac{1}{2 \pi i} \int_{C} \sum_{y=0}^{\infty} i \pi a^{w} w^{-k-1} 2^{-m-w+1} b^{\frac{1}{2}(-m-w-1)} e^{i \pi(2 y+1)(m+w)} d w \\
& =\frac{1}{2 \pi i} \int_{C} \pi a^{w} w^{-k-1}\left(-2^{-m-w}\right) b^{\frac{1}{2}(-m-w-1)} \csc (\pi(m+w)) d w
\end{aligned}
$$

from equation (1.232.3) in [5] where $\operatorname{csch}(i x)=-i \csc (x)$ from equation (4.5.10) in [1] and $\operatorname{Im}(w+m)>0$ for the sum to converge. 


\section{Definite integral in terms of the Lerch function}

Theorem 1. For $k, a, b \in \mathbb{C},-1<\operatorname{Re}(m)<-1 / 2$,

$$
\begin{aligned}
\int_{0}^{\infty} \frac{(\sqrt{b+x}-\sqrt{b})^{-m} \log ^{k}\left(\frac{a}{\sqrt{b+x}-\sqrt{b}}\right)}{x \sqrt{b+x}} d x & \\
= & (i \pi)^{k+1} e^{i \pi m} b^{-\frac{m}{2}-\frac{1}{2}} 2^{k-m+1} \Phi\left(e^{2 i m \pi},\right. \\
& \left.-k, \frac{i(-2 \log (a)+\log (b)+\log (4)-2 i \pi)}{4 \pi}\right)
\end{aligned}
$$

Proof. Since the right-hand side of equation (3) and (6) are equal we can equate the left-hand sides and simplify the factorials to get the stated result.

\section{Main results}

Proposition 1. For $-1<\operatorname{Re}(m)<-1 / 2,|\arg (b)|<\pi$,

$$
\begin{aligned}
& \int_{0}^{\infty} \frac{(\sqrt{b+x}-\sqrt{b})^{-m}}{x \sqrt{b+x}} d x \\
&=\pi\left(-2^{-m}\right) b^{-\frac{m}{2}-\frac{1}{2}} \csc (\pi m)
\end{aligned}
$$

Proof. Use equation (7) and set $k=0$ and simplify using entry (2) Table below $(64: 12: 7)$ in $[8]$.

Proposition 2. For $k \in \mathbb{C},-1<\operatorname{Re}(m)<-1 / 2$,

$$
\begin{aligned}
\int_{0}^{\infty} \frac{(\sqrt{x+1}-1)^{-m} \log ^{k}\left(\frac{2}{\sqrt{x+1}-1}\right)}{x \sqrt{x+1}} d x & \\
& =(i \pi)^{k+1} e^{i \pi m} 2^{k-m+1} \Phi\left(e^{2 i m \pi},-k, \frac{1}{2}\right)
\end{aligned}
$$

Proof. Use equation (7) and set $a=2, b=1$ and simplify.

Proposition 3. For $b \in \mathbb{C},-1<\operatorname{Re}(m)<-1 / 2$,

$\int_{0}^{\infty} \frac{(\sqrt{b+x}-\sqrt{b})^{-m} \log \left(\frac{\sqrt{b+x}+\sqrt{b}}{x}\right)}{x \sqrt{b+x}} d x=\pi 2^{-m-1} b^{-\frac{m}{2}-\frac{1}{2}} \csc (\pi m)(\log (4 b)+2 \pi \cot (\pi m))$ 
Proof. Use equation (7) and set $k=a=1$ and simplify using entry (3) Table below $(64: 12: 7)$ in $[8]$.

\section{Proposition 4.}

$$
\int_{0}^{\infty} \frac{\sqrt[3]{2}(\sqrt{4 x+1}-1)^{2 / 3}-\sqrt[4]{2}(\sqrt{4 x+1}-1)^{3 / 4}}{x \sqrt{4 x+1} \log \left(\frac{\sqrt{x+\frac{1}{4}}+\frac{1}{2}}{x}\right)} d x=\frac{1}{12} \log \left(\frac{1}{27}(99+70 \sqrt{2})\right)
$$

Proof. Use equation (7) and form a second equation by setting $m \rightarrow p$ and taking their difference. Next set $k=-1, a=1, b=1 / 4, m=-3 / 4, p=-2 / 3$ and simplify using entry (1) in Table below $(64: 12: 7)$ in [8].

\section{Proposition 5.}

$$
\int_{0}^{\infty} \frac{\sqrt[3]{2} \sqrt{\sqrt{4 x+1}-1}(\sqrt[6]{2}-\sqrt[6]{\sqrt{4 x+1}-1})}{x \sqrt{4 x+1} \log \left(\frac{\sqrt{4 x+1}+1}{2 x}\right)} d x=\log (3)
$$

Proof. Use equation (7) and form a second equation by setting $m \rightarrow p$ and taking their difference. Next set $k=-1, a=1, b=1 / 4, m=-1 / 2, p=-2 / 3$ and simplify using entry (1) in Table below $(64: 12: 7)$ in [8].

Theorem 2. For $k \in \mathbb{C},-1<\operatorname{Re}(m)<0$,

$$
\begin{aligned}
\int_{0}^{\infty} \frac{\left(\sqrt{x+\frac{1}{4}}-\frac{1}{2}\right)^{-m} \log ^{k}\left(-\frac{2}{\sqrt{4 x+1}-1}\right)}{x \sqrt{x+\frac{1}{4}}} d x & \\
& =2^{k+2}(i \pi)^{k+1} e^{-i \pi m} L i_{-k}\left(e^{2 i m \pi}\right)
\end{aligned}
$$

Proof. Use equation (7) and set $a=-1, b=1 / 4$ and simplify using equation (64:12:2) in $[8]$.

Theorem 3. For $k \in \mathbb{C}$,

$$
\int_{0}^{\infty} \frac{\sqrt{\sqrt{x+\frac{1}{4}}-\frac{1}{2}} \log ^{k}\left(-\frac{2}{\sqrt{4 x+1}-1}\right)}{x \sqrt{x+\frac{1}{4}}},-i^{k} 2^{k+2}\left(2^{k+1}-1\right) \pi^{k+1} \zeta(-k)
$$


Proof. Use equation (7) and set $a=-1, b=1 / 4, m=-1 / 2$ and simplify using entry (4) in Table below (64:12:7) and equation (64:12:1) and entry (2) in Table below (64:7) in [8].

\section{Proposition 6.}

$$
\int_{0}^{\infty} \frac{\left(\sqrt{x+\frac{1}{4}}-\frac{1}{2}\right)^{3 / 4}}{x \sqrt{x+\frac{1}{4}} \log ^{2}\left(-\frac{2}{\sqrt{4 x+1}-1}\right)} d x=-\frac{(1-i) C}{\sqrt{2} \pi}-\frac{\left(\frac{1}{48}+\frac{i}{48}\right) \pi}{\sqrt{2}}
$$

Proof. Use equation (13) and set $k=-2, m=-3 / 4$ and simplify in terms of Catalan's constant $(C)$ using equation (2.3) in [6] and section (1.7.6) in [4].

\section{Proposition 7.}

$$
\begin{aligned}
& \int_{0}^{\infty} \frac{\sqrt{\sqrt{x+\frac{1}{4}}-\frac{1}{2}} \log \left(\log \left(-\frac{2}{\sqrt{4 x+1}-1}\right)\right)}{x \sqrt{x+\frac{1}{4}} \log \left(-\frac{2}{\sqrt{4 x+1}-1}\right)} d x \\
& =\log (2)\left(2 i \gamma+\pi-i \log \left(8 \pi^{2}\right)\right)
\end{aligned}
$$

Proof. Use equation 14 and take the first partial derivative with respect to $k$ then apply L'Hopital's rule to the right-hand side as $k \rightarrow-1$ and simplify in terms of Euler's constant $(\gamma)$ using equation $(3: 10: 1)$ in [8] and (2.15) in [4].

\section{Proposition 8.}

$$
\int_{0}^{\infty} \frac{\sqrt{\sqrt{x+\frac{1}{4}}-\frac{1}{2}} \log \left(\log \left(-\frac{2}{\sqrt{4 x+1}-1}\right)\right)}{x \sqrt{x+\frac{1}{4}}} d x=\pi(\log (16)+i \pi)
$$

Proof. Use equation (14) and take the first partial derivative with respect to $k$ and set $k=0$ and simplify using equation $(3: 10: 1)$ in [8] and (2.15) in [4].

\section{Proposition 9.}

$$
\begin{aligned}
& \int_{0}^{\infty} \frac{\sqrt{\sqrt{x+\frac{1}{4}}-\frac{1}{2}} \log \left(-\frac{2}{\sqrt{4 x+1}-1}\right) \log \left(\log \left(-\frac{2}{\sqrt{4 x+1}-1}\right)\right)}{x \sqrt{x+\frac{1}{4}}} d x \\
& =\frac{1}{3} \pi^{2}(-3 \pi+2 i(-36 \log (A)+3+\log (128)+3 \log (\pi)))
\end{aligned}
$$

Proof. Use equation (14) and take the first partial derivative with respect to $k$ and set $k=1$ and simplify in terms of Glaisher's constant $(A)$ using equation $(3: 10: 1)$ in [8] and (2.15) in [4]. 


\section{Table of Integrals}

$$
\begin{aligned}
& f(x) \\
& \int_{0}^{\infty} f(x) d x \\
& \frac{(\sqrt{b+x}-\sqrt{b})^{-m}}{x \sqrt{b+x}} \\
& \pi\left(-2^{-m}\right) b^{-\frac{m}{2}-\frac{1}{2}} \csc (\pi m) \\
& \frac{(\sqrt{b+x}-\sqrt{b})^{-m} \log \left(\frac{\sqrt{b+x}+\sqrt{b}}{x}\right)}{x \sqrt{b+x}} \\
& \pi 2^{-m-1} b^{-\frac{m}{2}-\frac{1}{2}} \csc (\pi m)(\log (4 b)+2 \pi \cot (\pi m)) \\
& \frac{(\sqrt{x+1}-1)^{-m} \log ^{k}\left(\frac{2}{\sqrt{x+1}-1}\right)}{x \sqrt{x+1}} \\
& i \pi)^{k+1} e^{i \pi m} 2^{k-m+1} \Phi\left(e^{2 i m \pi},-k, \frac{1}{2}\right) \\
& \frac{\sqrt[3]{2}(\sqrt{4 x+1}-1)^{2 / 3}-\sqrt[4]{2}(\sqrt{4 x+1}-1)^{3 / 4}}{x \sqrt{4 x+1} \log \left(\frac{\sqrt{x+\frac{1}{4}}+\frac{1}{2}}{x}\right)} \\
& \frac{1}{12} \log \left(\frac{1}{27}(99+70 \sqrt{2})\right) \\
& \frac{\sqrt[3]{2} \sqrt{\sqrt{4 x+1}-1}(\sqrt[6]{2}-\sqrt[6]{\sqrt{4 x+1}-1})}{x \sqrt{4 x+1} \log \left(\frac{\sqrt{4 x+1}+1}{2 x}\right)} \\
& \log (3) \\
& \frac{\left(\sqrt{x+\frac{1}{4}}-\frac{1}{2}\right)^{-m} \log ^{k}\left(-\frac{2}{\sqrt{4 x+1}-1}\right)}{x \sqrt{x+\frac{1}{4}}} \\
& \frac{\left(\sqrt{x+\frac{1}{4}}-\frac{1}{2}\right)^{3 / 4}}{x \sqrt{x+\frac{1}{4}} \log ^{2}\left(-\frac{2}{\sqrt{4 x+1}-1}\right)} \\
& \frac{\sqrt{\sqrt{x+\frac{1}{4}}-\frac{1}{2}} \log ^{k}\left(-\frac{2}{\sqrt{4 x+1}-1}\right)}{x \sqrt{x+\frac{1}{4}}} \\
& \frac{\sqrt{\sqrt{x+\frac{1}{4}}-\frac{1}{2}} \log \left(\log \left(-\frac{2}{\sqrt{4 x+1}-1}\right)\right)}{x \sqrt{x+\frac{1}{4}} \log \left(-\frac{2}{\sqrt{4 x+1}-1}\right)} \\
& \frac{\sqrt{\sqrt{x+\frac{1}{4}}-\frac{1}{2}} \log \left(\log \left(-\frac{2}{\sqrt{4 x+1}-1}\right)\right)}{x \sqrt{x+\frac{1}{4}}} \\
& \frac{\sqrt{\sqrt{x+\frac{1}{4}}-\frac{1}{2}} \log \left(-\frac{2}{\sqrt{4 x+1}-1}\right) \log \left(\log \left(-\frac{2}{\sqrt{4 x+1}-1}\right)\right)}{x \sqrt{x+\frac{1}{4}}} \\
& 2^{k+2}(i \pi)^{k+1} e^{-i \pi m} \operatorname{Li}_{-k}\left(e^{2 i m \pi}\right) \\
& -\frac{(1-i) C}{\sqrt{2} \pi}-\frac{\left(\frac{1}{48}+\frac{i}{48}\right) \pi}{\sqrt{2}} \\
& i^{k} 2^{k+2}\left(2^{k+1}-1\right) \pi^{k+1} \zeta(-k) \\
& \log (2)\left(2 i \gamma+\pi-i \log \left(8 \pi^{2}\right)\right) \\
& \pi(\log (16)+i \pi) \\
& \frac{1}{3} \pi^{2}(-3 \pi+2 i(-36 \log (A)+3+\log (128)+3 \log (\pi)))
\end{aligned}
$$

\section{Discussion}

In this article we derived the definite integral of the logarithmic power square root of an algebraic function and expressed it in terms of the Lerch function. We then used this 
integral transform to derive a table of integrals consisting of mostly new formula in terms of special constants and special functions. The results presented were numerically verified for both real and imaginary values of the parameters in the integrals using Mathematica by Wolfram. In this work we used Mathematica software to numerically evaluate both the definite integral and associated Special function for complex values of the parameters $k, a, b, m$. We considered various ranges of these parameters for real, integer, negative and positive values. We compared the evaluation of the definite integral to the evaluated Special function and ensured agreement.

\section{Conclusion}

In this paper, we have derived a method for expressing definite integrals in terms of Special functions using our contour integration method. The contour we used was specific to solving integral representations in terms of the Lerch function. We expect that other contours and integrals can be derived using this method.

\section{Acknowledgements}

This research is supported by NSERC Canada under Grant 504070 .

\section{References}

[1] Milton Abramowitz and Irene A. Stegun. Handbook of Mathematical Functions: With Formulas, Graphs, and Mathematical Tables. Courier Corporation, 011965.

[2] Harry Bateman. Higher Transcendental Functions V.1. McGraw-Hill, 1953.

[3] Yu A. Brychkov, O. I. Marichev, and N. V. Savischenko. Handbook of Mellin Transforms. CRC Press, 102018.

[4] Steven R. Finch. Mathematical Constants ICM edition. Cambridge University Press, 072010.

[5] I. S. Gradshteyn and I. M. Ryzhik. Table of Integrals, Series, and Products. Academic Press, 052014.

[6] Leonard Lewin. Polylogarithms and Associated Functions. North Holland, 1981.

[7] M.A. Nyblom. On the evaluation of a definite integral involving nested square root functions. Rocky Mountain Journal of Mathematics, 37, 082007.

[8] Keith B. Oldham, Jan Myland, and Jerome Spanier. An Atlas of Functions: with Equator, the Atlas Function Calculator. Springer Science \& Business Media, 072010. 
[9] Robert Reynolds and Allan Stauffer. Definite integrals involving product of logarithmic functions and logarithm of square root functions expressed in terms of special functions. AIMS Mathematics, 5:5724-5733, 2020.

[10] Robert Reynolds and Allan Stauffer. A method for evaluating definite integrals in terms of special functions with examples. International Mathematical Forum, 15:235$244,2020$. 